\title{
Analysis of Multipath Routing-Part I: The Effect on the Packet Delivery Ratio
}

\author{
Aristotelis Tsirigos and Zygmunt J. Haas, Senior Member, IEEE
}

\begin{abstract}
In this paper, we develop an analytical framework for evaluating multipath routing in mobile ad hoc networks. The instability of the topology (e.g., failure of links) in this type of network due to nodal mobility and changes in wireless propagation conditions makes transmission of time-sensitive information a challenging problem. To combat the inherent unreliability of these networks, we propose a routing scheme that uses multiple paths simultaneously by splitting the information between a multitude of paths, so as to increase the probability that the essential portion of the information is received at the destination without incurring excessive delay.

Our scheme works by adding an overhead to each packet, which is calculated as a linear function of the original packet bits. The resulting packet (information and overhead) is fragmented into smaller blocks and distributed over the available paths. The probability of reconstructing the original information at the destination is derived in an analytical form and its behavior is studied for some special cases. It is shown that, under certain constraints, the packet dropping probability decreases as the number of used paths is increased.
\end{abstract}

Index Terms-Ad hoc networks, ad hoc routing, alternative path routing, diversity coding, multipath routing, network fault tolerance, quality of service.

\section{INTRODUCTION}

$\mathbf{I}$ $\mathrm{N}$ this paper, we consider the problem of routing data over multiple disjoint paths in an ad hoc network. A lot of research has been done in the area of multipath routing in wired networks. One of the initial approaches to this problem was the dispersity routing [1]. In [2], another multipath scheme is proposed, diversity coding, in order to achieve self-healing and fault tolerance in digital communication networks. In [3], a per-packet allocation granularity for multipath source routing schemes was shown to perform better than a per-connection allocation. An exhaustive simulation of the various tradeoffs associated with dispersity routing was presented in [4]. The inherent capability of this routing method to provide a large variety of services was pointed out. Due to this fact, numerous schemes employing multipath routing have been proposed for

Manuscript received February 20, 2002; revised October 3, 2002 and February 11, 2002; accepted February 23, 2003. The editor coordinating the review of this paper and approving it for publication is Y. Fang. This work was supported in part by the Department of Defense (DoD) Multidisciplinary University Research Initiative (MURI) program administered by the Office of Naval Research under Grant N00014-00-1-0564; in part by the DoD MURI program administered by the Air Force Office of Scientific Research under Grant F49620-02-1-0233; and in part by the National Science Foundation under Grant ANI-0081357.

A. Tsirigos is with the Courant Institute of Mathematical Sciences, New York University, New York, NY 10012 USA (e-mail: tsirigos@cs.nyu.edu).

Z. J. Haas is with the School of Electrical Engineering, Cornell University, Ithaca, NY 14853 USA (e-mail: haas@ece.cornell.edu).

Digital Object Identifier 10.1109/TWC.2003.821207 wired networks in order to perform quality of service (QoS) routing, such as in [5]-[12]. All these protocols are based on proactive routing, since they maintain tables that reflect the state of the entire network. Therefore, they cannot be successfully applied to mobile networks due to the unreliability of the wireless infrastructure and the nodal mobility, which can trigger an excessive amount of updates in the state tables.

The application of multipath techniques in mobile ad hoc networks seems natural, as multipath routing allows diminishing the effect of unreliable wireless links and the constantly changing topology. The on-demand multipath routing scheme is presented in [13] as a multipath extension of dynamic source routing (DSR) [14], in which alternate routes are maintained, so that they can be utilized when the primary one fails. Temporally ordered routing algorithm (TORA) [15], routing on-demand acyclic multipath (ROAM) [16] and ad hoc on-demand distance vector-backup routing (AODV-BR) [17], which is based on the ad hoc on-demand distance vector (AODV) protocol [18], are other examples of schemes that maintain multiple routes and utilize them only when the primary root fails. However, these protocols do not distribute the traffic into the multiple available paths. Another extension of DSR, multiple source routing (MSR) [19], proposes a weighted round-robin heuristic-based scheduling strategy among multiple paths in order to distribute load, but provides no analytical modeling of its performance. The split multipath routing (SMR), proposed in [20], focuses on building and maintaining maximally disjoint paths, however, the load is distributed only in two routes per session. In [21], the authors propose a novel and nearly linear heuristic for constructing a highly reliable path set. In [22], the positive effect of alternate path routing (APR) on load balancing and end-to-end delay in mobile ad hoc networks has been explored. It was argued, however, that the network topology and channel characteristics (e.g., route coupling) can severely limit the gain offered by APR strategies. In an interesting application [23], multipath path transport (MPT) is combined with multiple description coding (MDC) in order to send video and image information in a multihop mobile radio network.

In our paper, we propose a multipath scheme for mobile ad hoc networks based on diversity coding [2]. Data load is distributed over multiple paths in order to minimize the packet drop rate and achieve load balancing in a constantly changing environment. We derive an analytical expression of the probability that a transmission from the source results in successful packet reception and reconstruction at the destination for an arbitrary allocation of data to multiple paths. Our scheme's responsibility is to choose the data allocation that maximizes the probability of successful reception. 
The model we are using in order to evaluate our scheme is developed under the assumption that the mean time of packet transmission is much smaller than the mean time between variations in the network topology. If this assumption holds, then we can assume that the probability that one or more path links fail is constant during the transmission of a packet. In other words, one can assume that the topology of the network will not change significantly while a packet is being transmitted. We believe that this assumption is justified, because excessive variability in the network would mean that routes become obsolete before packets can even reach their destination, practically making route determination impossible.

Our paper is organized as follows. Section II provides a description of the proposed scheme and the definition of the successful transmission probability function $P_{\text {succ }}$, the function used for the evaluation of the scheme. In Section III, we derive an analytical formula for $P_{\text {succ }}$. $P_{\text {succ }}$ is analyzed for different cases and the optimal solution is calculated in an analytical form, whenever this is possible. Due to the fact that this is rarely so, an approximation of $P_{\text {succ }}$ is developed in [24]. Finally, in Section IV, we conclude this paper.

\section{DESCRIPTION OF OUR SCHEME}

In this section, we describe how our scheme exploits the multitude of paths, in order to offer increased protection against path failures. Data packets are sent from source to destination over these paths, making use of diversity coding [2], which we explain later in this section.

In our network model, we assume that $n_{\max }$ paths are available for the transmission of data packets from a source node to a destination node. Any of the multipath schemes mentioned in the introduction can be employed in order to acquire these paths. All paths are mutually disjoint; i.e., they have no nodes in common. Each path, indexed as $i, i=1, \ldots, n_{\max }$, is assigned a probability of failure $p_{i}$, which is the probability that path $i$ is down at the time that the source attempts to transmit. In addition, each path is treated as a pure erasure channel. Either no information reaches the destination through path $i$ (with probability $p_{i}$ ), or all the information is received correctly (with probability $1-p_{i}$ ). Since there are no common nodes among the paths, they are considered independent in the sense that success or failure of one path cannot imply success or failure of another. It should be noted here that in wireless ad hoc networks, nodes are sharing a single channel for transmission, so node disjointness does not guarantee the independence of the paths. Taking this into account, the paths in our paper are ideally considered independent as an approximation of a realistic ad hoc wireless network. For a more realistic modeling of the paths in a wireless network, one may refer to [24], where path correlation is included in our analysis.

Without loss of generality, the failure probabilities of the available paths are organized in the probability vector $p=\left[p_{i}\right], i=1 . . n_{\max }$, in such a way that $p_{i} \leq p_{i+1}$; i.e., the paths are ordered from the "best" one to the "worst" one. Given $\underline{p}$, we also define $\underline{q}=\left[q_{i}\right], q_{i}=1-p_{i}, i=1, \ldots, n_{\max }$, which is the vector of success probabilities. Throughout the paper, we use $\underline{p}$ and $\underline{q}$ interchangeably.
In [25], a path availability model for wireless ad hoc networks is proposed, which gives us some indication on how the quality of the network can be expressed in terms of the probability vector $p$. Also, there are other protocols, such as associativity-based routing (ABR) [26] and signal-stability-based routing (SSA) [27], that quantify the stability of the routes in a network using various criteria based on network measurements.

Let us suppose that the proposed scheme has to send a packet of $X$ information bits utilizing the set of available independent paths in such a way as to maximize the probability that these bits are successfully communicated to the destination. This probability is denoted as $P_{\text {succ }}$. In order to achieve this goal, we employ a coding scheme in which $Y$ extra bits are added as overhead. The resulting $B$ bits $(B=X+Y)$ are treated as one network-layer packet. The extra bits are calculated as a function of the information bits in such a way that, when splitting the $B$-bit packet into multiple equal-size nonoverlapping blocks, the initial $X$-bit packet can be reconstructed, given any subset of these blocks with a total size of $X$ or more bits. First, we define the overhead factor $r$

$$
r=\frac{B}{X}=\frac{b}{x}
$$

where $b$ and $x$ take integer values and the fraction $b / x$ cannot be further simplified; i.e., the greatest common divisor of $b$ and $x$ is 1 .

The key decision that we have to make is how the $B$ bits will be distributed over the available paths. For this reason, we define the vector $\underline{v}=\left[v_{i}\right]$, where $v_{i}$ is the number of equal-size blocks that is allocated to path $i$. Clearly, some of the paths may demonstrate such a poor performance that there is no point in using them at all. This means that we might require using only some of the available paths. If $n$ is the number of the paths we have to use in order to maximize $P_{\text {succ }}$, it would be preferable to define the block allocation vector $\underline{v}$ as a vector of a variable size $n$, instead of fixing its size to the number of available paths (i.e., $n_{\max }$ ). Given the fact that the probability vector is ordered from the best path to the worst one, a decision to use $n$ paths implies that these paths will be the first $n$ ones. Based on these observations, the allocation vector $\underline{v}$ has the following form:

$$
\underline{v}=\left(v_{1}, v_{2}, \ldots, v_{n}\right), n \leq n_{\max } .
$$

If the block size is $w$, then

$$
w \cdot \sum_{i=1}^{n} v_{i}=B=r X .
$$

Therefore, the total number of blocks that the $B$-bit packet is fragmented into is

$$
a=\sum_{i=1}^{n} v_{i}=\frac{r X}{w} .
$$

From $p_{i} \leq p_{i+1}$ follows that $v_{i} \geq v_{i+1}$, because a path with higher failure probability cannot be assigned fewer blocks than a path with a lower failure probability.

In Fig. 1, we can see the $B$-bit packet and its relation to the original $X$-bit packet (gray area). We also show how the $B$-bit packet is fragmented into equal-size nonoverlapping blocks of size $w$. The original $X$-bit packet is fragmented into 


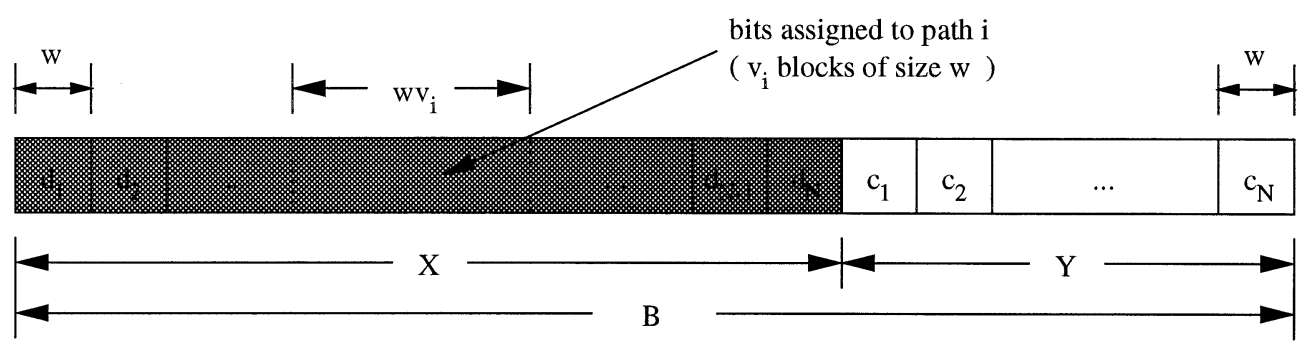

Fig. 1. Information and overhead packet fragmentation.

$N w$-size blocks, $d_{1}, \ldots, d_{N}$, and the $Y$-bit overhead packet into $M w$-size blocks, $c_{1}, \ldots, c_{M}$. Path 1 will be assigned the first $v_{1}$ blocks of the $B$-bit sequence, path two will receive the next $v_{2}$ blocks, and so on. Thus, path $i$ will be assigned $v_{i}$ blocks, each block of size $w$.

We can derive the expressions for $N$ and $M$ from Fig. 1

$$
\begin{aligned}
& N=\frac{X}{w}=\frac{a}{r} \\
& M=\frac{Y}{w}=(r-1) N=\frac{r-1}{r} a .
\end{aligned}
$$

This is a typical case where $M$-for- $N$ diversity coding can be applied. In [2], Ayanoglu et al. have proved that if $M$ or fewer blocks are lost, out of the $N+M$ total data and overhead blocks, the original $N$ information blocks can be recovered using appropriate linear transformations. The overhead blocks $c_{i}, i=1, \ldots, M$ are also calculated as a linear transformation of the information blocks $d_{i}, i=1, \ldots, N$. The block size has to satisfy the following inequality, so that the original information can be recovered:

$$
w \geq\left\lceil\log _{2}(N+M+1)\right\rceil \geq \log _{2}(a+1)
$$

where we used (5) and (6). By incorporating (4) into (7), we obtain an inequality for the number of blocks $a$, into which we can split the $B$-bit packet

$$
B \geq a \log _{2}(a+1) \equiv B_{\min } .
$$

In Fig. 2, we plot the minimum value of $B$ (i.e., $B_{\min }$ ) against the total number of blocks, i.e., the minimum required size of the packet in bits, as a function of the number of blocks we want to split it into.

The optimization algorithm we will develop in the following section is used to determine the optimal number of paths and the optimal allocation vector, given the path probability vector $\underline{p}$ and the overhead factor $r$. The optimization process involves the maximization of $P_{\text {succ }}$, the definition of which we give shortly.

If $v_{i}$ is the number of blocks we send over path $i$, and $z_{i}$ the number of blocks that actually reach the destination through path $i$, then

$$
\begin{array}{r}
\operatorname{Pr}\left\{z_{i}=v_{i}\right\}=q_{i} \\
\operatorname{Pr}\left\{z_{i}=0\right\}=p_{i}
\end{array}
$$

because we assume that if a path fails, then all the blocks sent over the path are lost (recall the pure erasure channel assumption). $M$-for- $N$ diversity coding can reconstruct the original $X$-bit information packet, provided that at least $N$ blocks reach

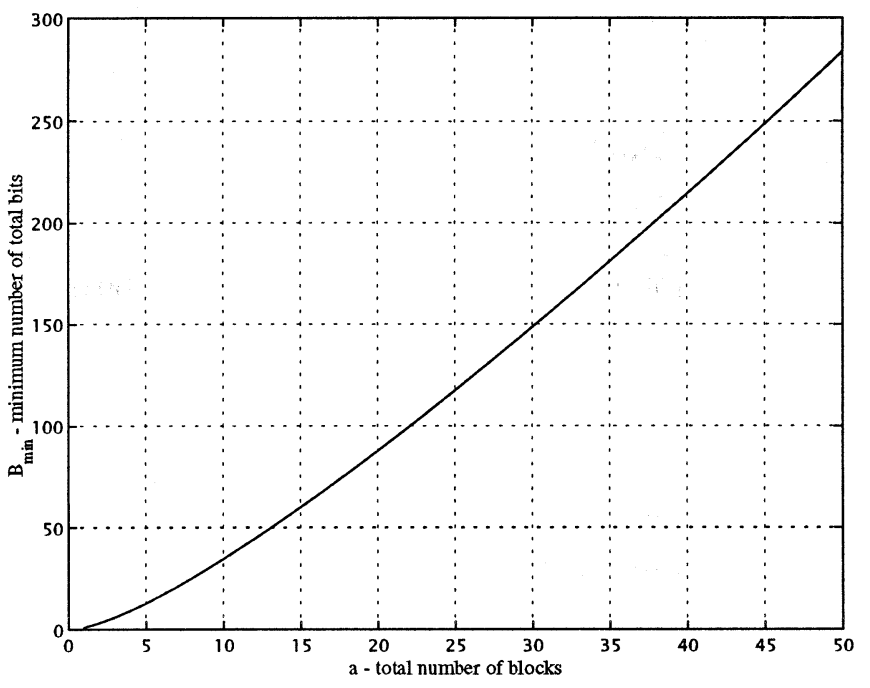

Fig. 2. Minimum number of total bits versus total number of blocks.

the destination. Therefore, we can define $P_{\text {succ }}$ in terms of the number of paths that are actually used and the corresponding allocation vector

$$
P_{\text {succ }}(n, \underline{v})=\operatorname{Pr}\left\{\sum_{i=1}^{n} z_{i} \geq \frac{a}{r}\right\}
$$

where we expressed $N$ as a function of $a$ and $r$, using (5).

\section{Evaluation Function $P_{\text {succ }}$}

In this section, we use the definition in (9) in order to provide an analytical formula for $P_{\text {succ }}$ and its complexity (Section III-A). In Section III-B, we propose the use of a uniform block allocation, i.e., one block per path, in order to maximize $P_{\text {succ }}$. In Section III-C, the uniform block allocation case is analyzed, and finally, results are presented for the special case in which all paths have the same failure probability $p_{i}=p$, $i=1, \ldots, n$ (Section III-D). Only in the last case we are able to provide an analytical solution, i.e., the optimal number of paths.

\section{A. Formula and Complexity of $P_{\text {succ }}$}

In this section, we present a formula for the calculation of the probability of success $P_{\text {succ }}$, given the probability vector $p$, the overhead factor $r$, and the allocation vector $\underline{v}$. We also give an estimation of the complexity of this function in terms of the number of the multiplications involved in its calculations.

According to our network model, each one of the $n$ paths used by our scheme is subject to two distinct events: 
- the event of failure to transmit the assigned packets (probability $p_{i}$ );

- the event of successful attempt to transmit the packets (probability $q_{i}$ ).

We define an $n$-dimensional vector $\underline{s}$, which reflects the state of the $n$ paths:

- $s_{i}=0$, if path $i$ failed;

- $s_{i}=1$, if path $i$ succeeded.

The associated probabilities are:

- $\operatorname{Pr}\left\{s_{i}=0\right\}=p_{i}$

- $\operatorname{Pr}\left\{s_{i}=1\right\}=q_{i}=1-p_{i}$.

The probability $t(\underline{s})$ of the $n$ paths being in state $\underline{s}$ is easily calculated as

$$
t(\underline{s})=\prod_{i=1}^{n} p_{i}{ }^{1-s_{i}} q_{i}{ }^{s_{i}}
$$

The cost of calculating $t(\underline{s})$, defined as the number of multiplication operations, is

$$
C\{t(\underline{s})\}=n .
$$

Each different state corresponds to a different set of paths succeeding in transmitting packets. All possible states describe all combinations of such sets, thus covering the whole space of events ( $2^{n}$ events in total). A transmission is successful if and only if at least $N$ blocks arrive at the destination. Consequently, each term $t(\underline{s})$, defined in (10), can contribute to $P_{\text {succ }}$ only if the number of blocks sent over the set of paths described by $s$ is more than or equal to $N$. By making this observation and by replacing $N$ using (5), we can write $P_{\text {succ }}$ as $^{1}$

$$
P_{\text {succ }}(n, \underline{v})=\sum_{\underline{s}} t(\underline{s}) \cdot u\left(\underline{s} \cdot \underline{v}-\frac{a}{r}\right)
$$

where $\underline{s} \cdot \underline{v}$ is the inner product of vectors $\underline{s}$ and $\underline{v}$, and equals the total number of successfully received blocks allocated to the subset of paths described by the state vector $\underline{s}$. The function $u(\cdot)$ in (12) is the unit step function defined as

$$
u(x)= \begin{cases}0, & x<0 \\ 1, & x \geq 0\end{cases}
$$

The cost in multiplication operations of calculating $P_{\text {succ }}$ using (12) is

$$
C\left\{P_{\text {succ }}(n, \underline{v})\right\}=n \cdot 2^{n}
$$

because the total number of states is $2^{n}$ and each term has a cost of $n$ [see (11)]. Given the probability vector $p$ and the overhead factor $r$ as parameters, we are looking for the optimal number $n_{\text {opt }}$ of paths to use (out of the $n_{\max }$ available ones) as well as the optimal allocation vector $\underline{v}_{\text {opt }}$ over the $n_{\text {opt }}$ paths, so that $P_{\text {succ }}$ is maximized.

Equation (12) is a complex formula, which makes it impossible to apply analytical maximizing techniques such as Lagrange multipliers, primarily because of the presence of the unit step function. Let us estimate the cost of exhaustively testing all combinations of the allocation vector $\underline{v}$, in order to find the maximum of $P_{\text {succ }}$. The number of all possible allocations of $B$ bits

\footnotetext{
${ }^{1}$ The reader is reminded that $a=\sum_{i=1}^{n} v_{i}$.
}

to $n$ paths is $\left(\begin{array}{c}B+n \\ n\end{array}\right)$. As we have explained before, for a given $n$ and $\underline{v}$, computing $P_{\text {succ }}$ involves $n \cdot 2^{n}$ multiplication operations. Therefore, the total cost is

$$
C=\sum_{n=1}^{n_{\max }} n 2^{n}\left(\begin{array}{c}
B+n \\
n
\end{array}\right) .
$$

It is clear that the cost of brute-force optimization of $P_{\text {succ }}$ is exponential. Even for a small number of paths (e.g., 10 paths), testing all the valid combinations will take an unacceptably long time, making the optimization of $P_{\text {succ }}$ impossible to implement in real time. The reader is reminded that in a mobile ad hoc network, the probability vector $p$ will not be constant with time, and therefore, the optimization process of $P_{\text {succ }}$ must be repeated when the network topology changes.

\section{B. Analyzing $P_{\text {succ }}$}

In Section II, we showed that $P_{\text {succ }}$, as a function of the number of used paths and the allocation vector $\underline{v}$, consists of $2^{n}$ different terms, each one associated with a different state. The network states can be ordered into $n$ classes according to the number $k$ of successful path transmissions involved in each one, i.e., the number of 1's in each state vector, or equivalently, the number of $q_{i}$ 's in the associated probability factor $t(\underline{s})$. What follows is the definition of the $k$-order class of states, denoted as $S_{k}$ :

$$
S_{k}=\left\{\underline{s}: \sum_{i=1}^{n} s_{i}=k\right\} .
$$

The size of class $S_{k}$ is $\left|S_{k}\right|=\left(\begin{array}{l}n \\ k\end{array}\right)$. Given the classification of states with respect to the number of successful transmission attempts, we can alter (12) and express $P_{\text {succ }}$ as a double sum over the $n$ different classes of states and the $\left|S_{k}\right|$ terms of each class

$$
P_{\text {succ }}(n, \underline{v})=\sum_{k=1}^{n} \sum_{\underline{s} \in S_{k}} t(\underline{s}) \cdot u\left(\underline{s} \cdot \underline{v}-\frac{a}{r}\right) .
$$

Because of the complexity of $P_{\text {succ }}$, we will maximize it assuming a uniform block allocation; i.e., one block is sent over each path. The maximization algorithm will be presented in Section IV. In [24], we will develop a technique in order to approximate the optimal block allocation without assuming a uniform allocation. Here, we just derive the simplified expression of $P_{\text {succ }}$ when one block is sent per path

$$
P_{\text {succ }}(n, \underline{v})=\sum_{k=1}^{n} \sum_{\underline{s} \in S_{k}} t(\underline{s}) \cdot u\left(k-\frac{a}{r}\right) .
$$

By observing that the unit step function in the previous equation yields one when $k \geq n / r$, we can rewrite (17) as

$$
P_{\text {succ }}(n, \underline{v})=\sum_{k=m}^{n} \sum_{\underline{s} \in S_{k}} t(\underline{s})
$$

where

$$
m=\left\lceil\frac{n}{r}\right\rceil .
$$




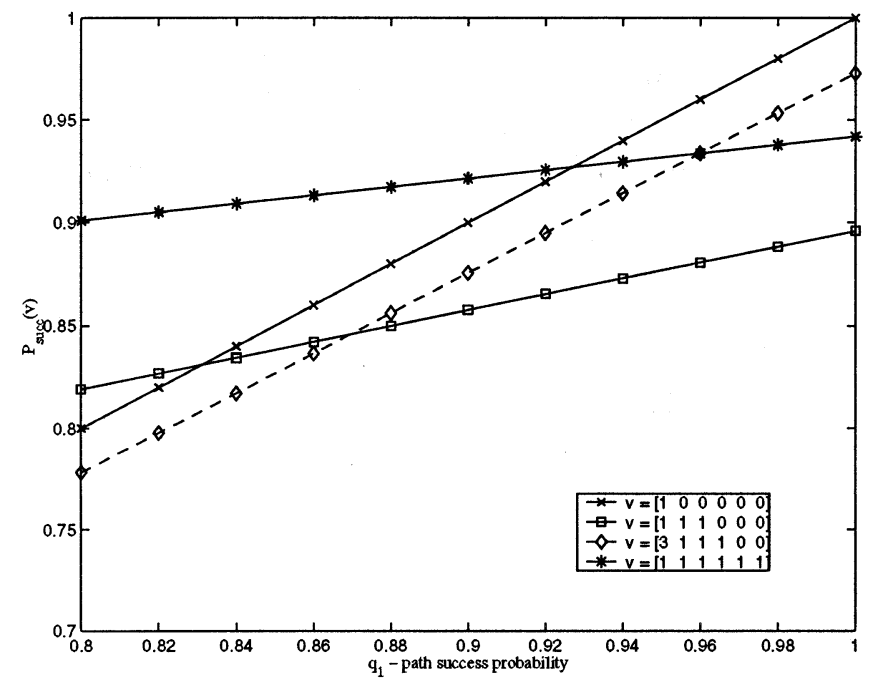

Fig. 3. Comparing different allocation vectors.

If $n / r$ is not an integer, then the "effective" overhead factor $r^{\prime}$ is

$$
r^{\prime}=\frac{n}{m}<r
$$

Therefore, in order to make complete use of the overhead factor $r, m$ has to take integer values and, of course, at the same time $n$ has to be an integer itself. The combination of these two requirements yields the potential maximal points of $P_{\text {succ }}$

$$
n=k \cdot b
$$

where $b$ is defined in (1).

In Fig. 3, we give a numerical example in the case where $r=3 / 2$ and $n_{\max }=6$ paths. The probability vector is $\underline{q}=$ $\left[q_{1}, 0.8,0.8,0.8,0.8,0.8\right]$, where $0.8 \leq q_{1} \leq 1$. We plot the probability of success for four allocation vectors, as shown in the legend of the graph. We can see that for $q_{1}<0.92$, the optimal allocation is $\underline{v}=[1,1,1,1,1,1]$, whereas for $q_{1}>$ 0.92 , it is $\underline{v}=[1]$. If only three paths are available, then for $q_{1}<0.83$, the optimal allocation is $\underline{v}=[1,1,1]$, whereas for $q_{1}>0.83$, it is $\underline{v}=[1]$. We also verify that the vector $\underline{v}=$ $[3,1,1,1,1]$ can never be the optimal.

\section{Uniform Block Allocation}

The goal of this section is to explore the behavior of $P_{\text {succ }}$ as the number of paths $n$ increases, assuming a uniform block distribution; i.e., each path is assigned one block $\left(v_{i}=1, i=\right.$ $1, \ldots, n)$.

We start by giving some more definitions. The function $T_{k}(n)$ is the sum of terms of all states $\underline{s}$ that belong to class $S_{k}$ when $n$ paths are being used

$$
T_{k}(n)=\sum_{\underline{s} \in S_{k}} t(\underline{s}), \quad 0 \leq k \leq n .
$$

We also define the sum of all terms from class $k$ and up

$$
T_{k}^{\prime}(n)=\sum_{j=k}^{n} T_{j}(n)=\sum_{l=k}^{n} q_{l} T_{k-1}(l-1) .
$$

The first sum represents the sum of terms of class $k$ and up, and, as it is proved in the Appendix, it is equal to the second sum, which only includes terms of class $k$, making it much more attractive in terms of computational cost. Using (22) and (23), the expression for $P_{\text {succ }}$ found in (18) can be written as

$$
P_{\text {succ }}^{(u)}(n)=T_{m}^{\prime}(n)
$$

where $m=\lceil n / r\rceil$ [see (19)]. The computation cost, measured by the number of multiplications, is

$$
C\left\{P_{\text {succ }}^{(u)}(n)\right\}=\sum_{k=m}^{n} k\left(\begin{array}{c}
k-1 \\
m-1
\end{array}\right)=m \sum_{k=m}^{n} k\left(\begin{array}{c}
k \\
m
\end{array}\right) .
$$

It is interesting to observe the behavior of $P_{\text {succ }}$ as $n$ increases. In order to do this, we first define the following function:

$$
d m(n)=m(n)-m(n-1)=\left\lceil\frac{n}{r}\right\rceil-\left\lceil\frac{(n-1)}{r}\right\rceil .
$$

Note that $d m$ is a periodic function with period $b$ [see (1)]. The proof can be found in the Appendix. Our goal is to calculate $d P_{\text {succ }}$, which is the gain in probability of success $P_{\text {succ }}$ when moving from $n-1$ paths to $n$ paths

$$
d P_{\text {succ }}^{(u)}(n)=P_{\text {succ }}^{(u)}(n)-P_{\text {succ }}^{(u)}(n-1) .
$$

We distinguish two different cases, according to the value of $d m$ :

$$
\begin{aligned}
& d m(n)=0: \\
& d P_{\text {succ }}^{(u)}(n)=q_{n} T_{m-1}^{\prime}(n-1)>0 \\
- & d m(n)=1: \\
& d P_{\text {succ }}^{(u)}(n)=-p_{n} T_{m-1}^{\prime}(n-1)<0 .
\end{aligned}
$$

The inequalities (28) and (29), for which a proof is found in the Appendix, show that the evaluation function exhibits local maxima at positions $n_{0}$, if $d m\left(n_{0}\right)=0$ and $d m\left(n_{0}+1\right)=1$.

In order to demonstrate the behavior of $P_{\text {succ }}$, we provide an example for $n_{\max }=20$ paths. In Figs. 4 and 5, we plot $P_{\text {succ }}$ and $d m$. In the first figure, the overhead factor is $r=3 / 2$ and the path success probability for all paths is $q=0.8$, whereas in the second one, $r=2$ and $q=0.7$. One can verify that all local maxima are encountered at positions $n_{0}$, where $d m\left(n_{0}\right)=0$ and $d m\left(n_{0}+1\right)=1$.

\section{Uniform Probability Vector Case}

In this section, we will provide an analysis for the special case in which all paths have the same probability of success $q$. A uniform block allocation is assumed. We show that, if $q$ is greater than or equal to a threshold value, then $P_{\text {succ }}$, evaluated only in the subspace of its local maxima (positions $n=k \cdot b, k$ integer), is an ascending function with respect to $n$.

In the case of uniform path probability and uniform block allocation, we can write $P_{\text {succ }}$ as

$$
P_{\text {succ }}^{(u)}(n)=\sum_{i=m}^{n} c_{i, n}
$$




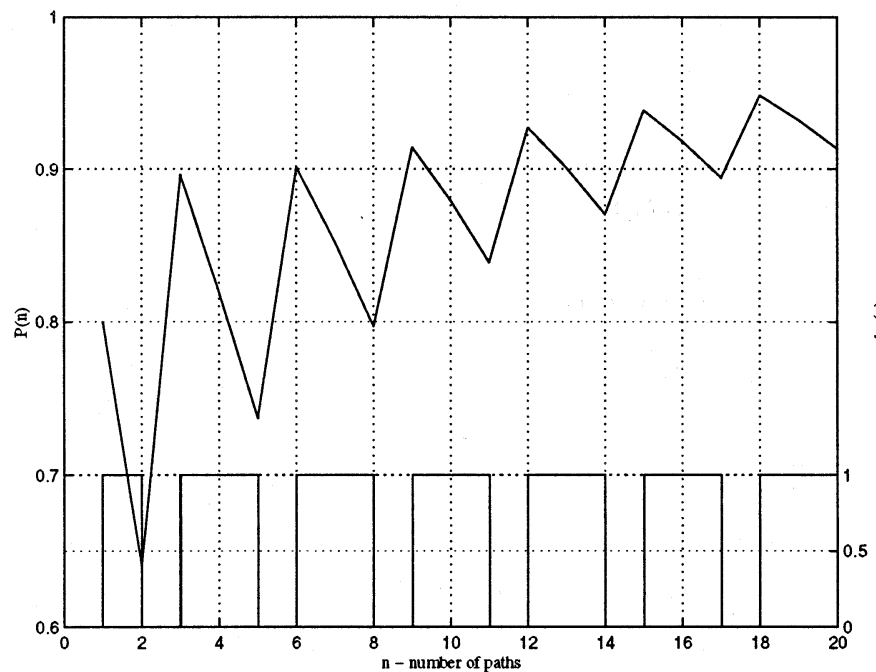

Fig. 4. Local maxima for $r=3 / 2$ and $q=0.8$.

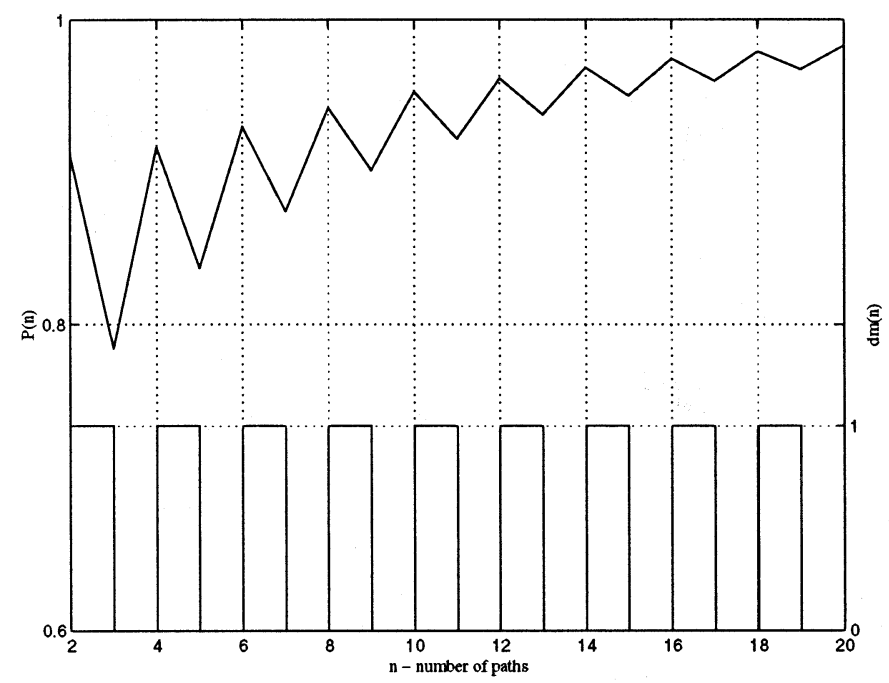

Fig. 5. Local maxima for $r=2$ and $q=0.7$.

where $m=\lceil n / r\rceil$. The terms $c_{i, n}$ for $0 \leq i \leq n$ are given by the following equation:

$$
c_{i, n}=\left(\begin{array}{c}
n \\
i
\end{array}\right) q^{i} p^{n-i}
$$

where $p=1-q$. In Fig. 6, we have plotted the terms $c_{i, n}$, for $0 \leq i \leq n$. The sum of these terms, $\sum_{i=0}^{n} c_{i, n}$, is 1 , and as we increase $n$, the area under the curve that connects the successive values $c_{i, n}$ approaches the value of the sum, i.e., it approaches 1.

The area close to the tails of the graph in Fig. 6 approaches zero exponentially as $n$ increases. Consequently, as $n$ increases, the total area under the curve is concentrated in the proximity of the maximum point of the curve, which is found at position $i_{0}$

$$
i_{0}=n q-p .
$$

In order to calculate the sum of the terms $c_{i, n}$ for $m \leq i \leq n$, or alternatively, the corresponding area under the curve (provided that $n$ is sufficiently large), we have to find if the area of interest

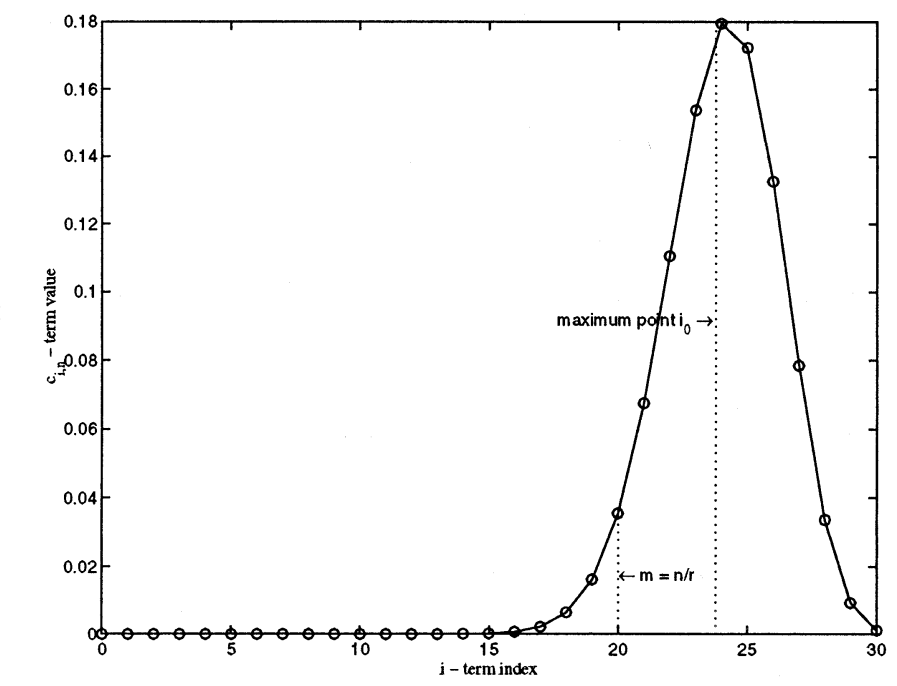

Fig. 6. Values of terms $c_{i, n}$, for $0 \leq i \leq n$.

TABLE I

THRESHOLDS $q_{0}$ AND $q_{+}$FOR

$r<2$

\begin{tabular}{ccc}
$r$ & $q_{0}(r)$ & $q_{+}(r)$ \\
\hline $5 / 4$ & 0.80 & 0.88
\end{tabular}

$4 / 3 \quad 0.75 \quad 0.84$

$\begin{array}{lll}3 / 2 & 0.67 & 0.79\end{array}$

$\begin{array}{lll}5 / 3 & 0.60 & 0.68\end{array}$

$7 / 4 \quad 0.57 \quad 0.63$

$9 / 5 \quad 0.56 \quad 0.61$

contains the maximum or not. This is done by studying the behavior of $m$ and $i_{0}$, as $n$ increases

$$
\begin{aligned}
& \frac{d i_{0}(n)}{d n}=q \\
& \frac{d m(n)}{d n}=\frac{1}{r} .
\end{aligned}
$$

It is easily concluded that the maximum point lies inside the area of interest (eventually, for large values of $n$ ), only if $q>1 / r \equiv$ $q_{0}$. Thus, we derive the "basic" threshold $q_{0}$, according to which

$$
\lim _{n \rightarrow+\infty} P_{\text {succ }}^{(u)}(n)= \begin{cases}0, & q<q_{0} \\ 1, & q>q_{0} .\end{cases}
$$

Also, if $q>q_{0}$, there is a value of $n$ beyond which $P_{\text {succ }}$ is ascending within the subset of the potential global maxima, which are found at $n=k \cdot b, k \geq 1$. Thus, we define a new threshold, $q_{+}$, so that for all $q \geq q_{+}$, function $P_{\text {succ }}$ is ascending for all $k \geq 1$, and for all $q<q_{+}$, it is not. This threshold can be calculated as the value of $q$ for which the first two local maxima, $n=b$ and $n=2 b$, for $k=1$ and $k=2$, are equal

$$
\sum_{i=x}^{b}\left(\begin{array}{c}
b \\
i
\end{array}\right) q^{i} p^{b-i}=\sum_{i=2 x}^{2 b}\left(\begin{array}{c}
2 b \\
i
\end{array}\right) q^{i} p^{b-i} .
$$




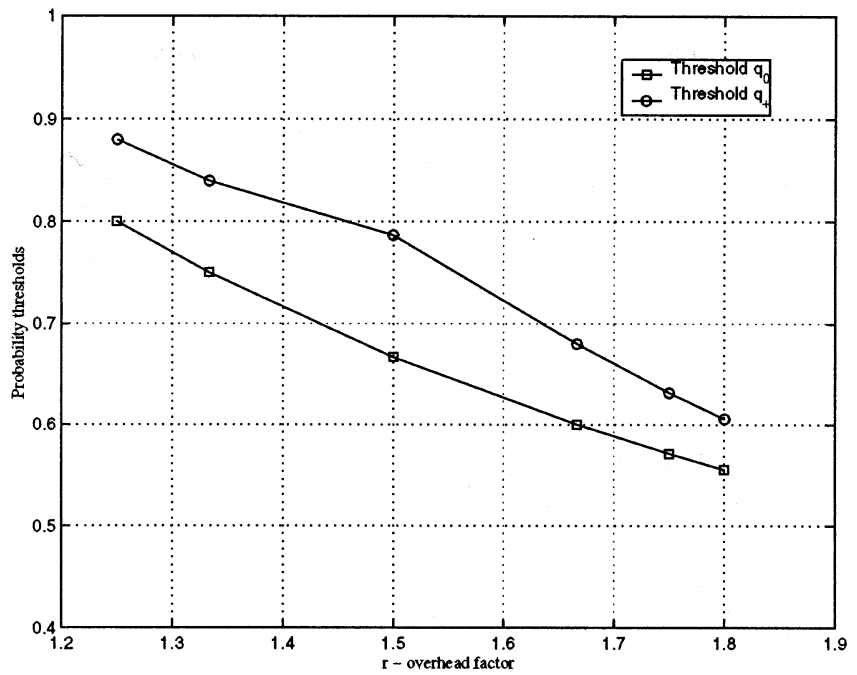

Fig. 7. Thresholds $q_{0}$ and $q_{+}$for $r<2$.

In Table I, $q_{0}$ and $q_{+}$are computed for various values of $r<2$. In Fig. 7, we plot the results listed in the table.

In summary, in this section, we derived two different thresholds, $q_{0}$ and $q_{+}$. If $q<q_{0}$, then $P_{\text {succ }}$ is descending within the set of its local maxima. In contrast, if $q>q_{+}$, then $P_{\text {succ }}$ is ascending within the same set. If $q_{0} \leq q \leq q_{+}$, then $P_{\text {succ }}$ is descending until a minimum point, and then it is ascending.

We will proceed by giving an idea of the maximum probability gain offered by our scheme. Due to the limited bandwidth of wireless networks, it seems reasonable to require that $r$ is less than two, so that the traffic load is increased by less than $100 \%$. However, we shall leave this up to the specific application, since increased reliability may be required, even at the expense of higher traffic load. The probability gain is defined as the increase in $P_{\text {succ }}$ when using multiple paths, as compared with only one path is used. In Fig. 8, we show the $P_{\text {succ }}$ yielded by our scheme versus the path success probability $q$ for $n_{\max }=$ 10, 20, and 30 paths. From Fig. 9, it is obvious that we can increase $P_{\text {succ }}$ by more than $25 \%$, provided that enough paths are available.

\section{CONCLUSION}

In this paper, we proposed a new scheme for multipath routing in mobile ad hoc networks. Our goal was to apply multipath techniques in an environment that has continuously changing topology and no infrastructure, so that the typical problems associated with nodal mobility and wireless links (unreliable transmissions, fading, etc.) can be alleviated. We argued that if the mean time of packet transmission is much smaller than the mean time between variations in network topology, we can fairly assume that the probability that one or more path links fail is constant during the transmission of a packet.

Under these assumptions, we considered the general case of multipath transmission, in which $n_{\max }$ disjoint paths are available for a packet transmission. Each path is treated as a pure erasure channel, and it is associated with some failure probability

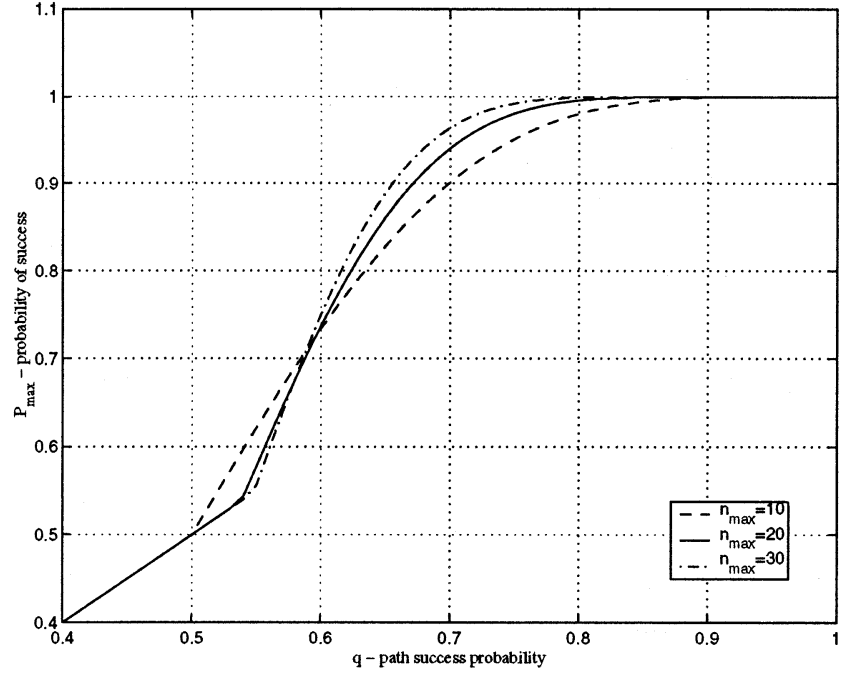

Fig. 8. Probability of successful transmission $\left(P_{\text {succ }}\right)$.

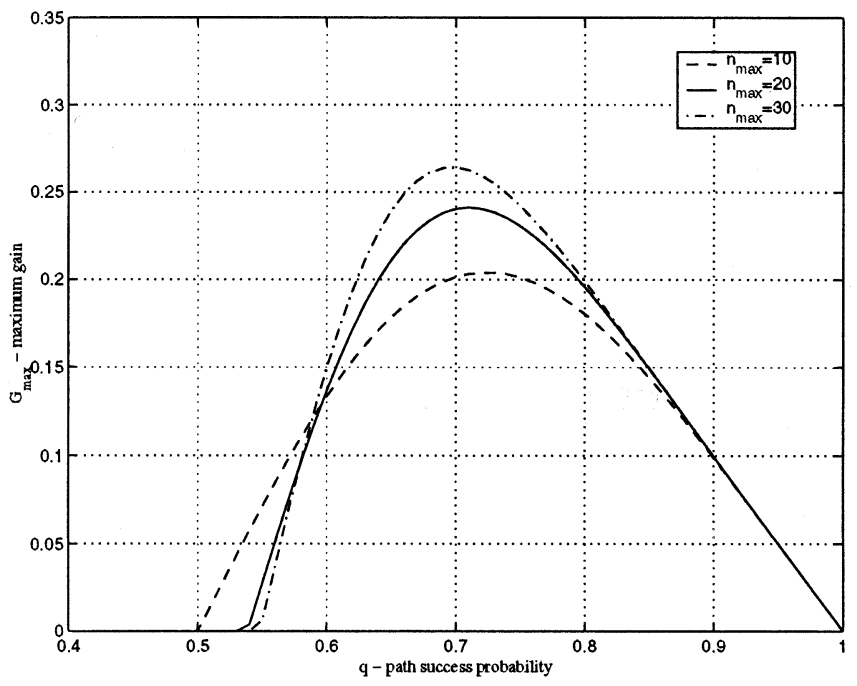

Fig. 9. Probability gain.

$p_{i}$, which was defined as the probability that, at transmission attempt time, the path is down. Based on the work done in [2], we used $M$-for- $N$ diversity coding. This scheme splits the original packet into $N$ blocks, adds $M$ blocks of overhead (calculated using linear transformations from the original $N$ blocks), and finally, allocates one block to each one of $N+M$ paths. The $M$-for- $N$ diversity coding offers protection against, at most, $M$ lost blocks out of the total $N+M$ blocks. In our scheme, rather than allocating one block per path, we assume an allocation of $v_{i}$ blocks to path $i, i=1, \ldots, n_{\max }$. Thus, we show what the optimal distribution of these blocks to the $n_{\max }$ disjoint paths should be, so that $P_{\text {succ }}$ is maximized.

Given the path failure probabilities, the overhead factor, and the allocation of the original and overhead blocks to the $n_{\max }$ paths, we developed an analytical formula for the probability function $P_{\text {succ }}$, namely, the probability that no more than $M$ blocks are lost. This is the probability that the original $N$ blocks can be reconstructed at the destination, and, as a consequence, the transmission is successful. In the case where all the paths 
have the same probability of success, we studied the behavior of $P_{\text {succ }}$ as the number of utilized paths increases.

Our scheme proposed here offers increased protection against route failures. Compared with simple packet replication, it can improve the successful transmission probability by up to $25 \%$. Under some constraints on the path failure probabilities, it was found that the probability of a successful communication of packets between source and destination increases with the number of used paths. Improved delivery ratio combined with load balancing (achieved because the optimal solution tends to distribute bits equally among the paths) may tend to reduce the end-to-end delay, however, increased traffic load due to diversity coding overhead might cancel out the benefits. Future work could address the problem of modeling the effect of multipath routing on the end-to-end delay, taking all factors into account.

So far, we intentionally left out of our discussion an important implementation aspect of our scheme. Although we offer a method for the optimal usage of multiple paths, we do not suggest any specific way for tuning the optimization process with respect to the frequency with which it is applied. Ideally, the optimal solution should be recalculated whenever a significant change in the path probabilities is detected. To this end, we developed a fast polynomial-time algorithm. A discussion about possible update strategies and their evaluation is an interesting topic, but is out of scope of our paper, since it would require modeling of additional parameters, such as mobility patterns, computational power, etc.

In [24], we develop an analytical approximation of $P_{\text {succ }}$, that enables us to maximize it in the case where the probability vector is not uniform. The optimization process is performed in polynomial time. There, we also extend our path model, so as to allow correlation among the available paths, and we provide a simple heuristic method in order to find a suboptimal solution to the maximization problem.

\section{APPENDIX}

\section{Period of Function $d m$}

In this section, we prove that $d m$ is a periodic function with period $b$, which is defined in (1)

$$
\begin{aligned}
d m(n+b) & =\left\lceil\frac{n+b}{r}\right\rceil-\left\lceil\frac{n+b+1}{r}\right\rceil \\
& =\left\lceil\frac{n}{r}+\frac{b}{\frac{b}{x}}\right\rceil-\left\lceil\frac{n-1}{r}+\frac{b}{\frac{b}{x}}\right\rceil \\
& =\left\lceil\frac{n}{r}+x\right\rceil-\left\lceil\frac{n-1}{r}+x\right\rceil \\
& =d m(n) .
\end{aligned}
$$

Proof of (23), (28), and (29)

We have to prove that

$$
T_{k}^{\prime}(n)=\sum_{j=k}^{n} T_{j}(n)=\sum_{l=k}^{n} q_{l} T_{k-1}(l-1) .
$$

At first, we observe that both sums yield $q_{1}$ for $n=1$. Since we know that

$$
P_{\text {succ }}^{(u)}(n)=T_{m}^{\prime}(n)
$$

it is sufficient to prove (28)and (29) for both sums of (23). For $d m(n)=0$, we show the following:

- First, we calculate $d P_{\text {succ }}^{(u)}$ using the first sum in (23)

$$
\begin{aligned}
d P_{\text {succ }}^{(u)}(n)= & \sum_{k=m}^{n} T_{k}(n)-\sum_{k=m}^{n-1} T_{k}(n-1) \\
= & \sum_{k=m}^{n} p_{n} T_{k}(n-1)+q_{n} T_{k-1}(n-1) \\
& -\sum_{k=m}^{n-1} T_{k}(n-1) \\
= & p_{n} \sum_{k=m}^{n-1} T_{k}(n-1)+q_{n} \sum_{k=m-1}^{n-1} T_{k}(n-1) \\
& -\sum_{k=m}^{n-1} T_{k}(n-1) \\
= & q_{n} T_{m-1}(n-1)+\left(p_{n}+q_{n}-1\right) \\
& \times \sum_{k=m}^{n-1} T_{k}(n-1) \\
= & q_{n} T_{m-1}(n-1) .
\end{aligned}
$$

- We get the same result if we use the second sum in (23)

$$
\begin{aligned}
d P_{\text {succ }}^{(u)}(n) & =\sum_{l=m}^{n} q_{l} T_{m-1}(l-1)-\sum_{l=m}^{n-1} q_{l} T_{m-1}(l-1) \\
& =q_{n} T_{m-1}(n-1) .
\end{aligned}
$$

For $d m(n)=1$, we have the following:

- The first sum yields

$$
\begin{aligned}
d P_{\text {succ }}^{(u)}(n)= & \sum_{k=m}^{n} T_{k}(n)-\sum_{k=m-1}^{n-1} T_{k}(n-1) \\
= & \sum_{k=m}^{n} p_{n} T_{k}(n-1)+q_{n} T_{k-1}(n-1) \\
& -\sum_{k=m-1}^{n-1} T_{k}(n-1) \\
= & p_{n} \sum_{k=m}^{n-1} T_{k}(n-1)+q_{n} \sum_{k=m-1}^{n-1} T_{k}(n-1) \\
& -\sum_{k=m-1}^{n-1} T_{k}(n-1) \\
= & -p_{n} T_{m-1}(n-1)+\left(p_{n}+q_{n}-1\right) \\
& \times \sum_{k=m-1}^{n-1} T_{k}(n-1) \\
= & -p_{n} T_{m-1}(n-1) .
\end{aligned}
$$


- The second sum gives the same result

$$
\begin{aligned}
d P_{\text {succ }}^{(u)}(n)= & \sum_{l=m}^{n} q_{l} T_{m-1}(l-1)-\sum_{l=m-1}^{n-1} q_{l} T_{m-2}(l-1) \\
= & \sum_{l=m}^{n} q_{l} T_{m-1}(l-1) \\
& -\sum_{l=m-1}^{n-1}\left[T_{m-1}(l)+p_{l} T_{m-1}(l-1)\right] \\
= & p_{n} \sum_{k=m}^{n-1} T_{k}(n-1) \\
& +q_{n} \sum_{k=m-1}^{n-1} T_{k}(n-1)-\sum_{k=m-1}^{n-1} T_{k}(n-1) \\
= & -p_{n} T_{m-1}(n-1) \\
& +\sum_{l=m}^{n}\left(q_{l}-1+p_{l}\right) T_{m-1}(l-1) \\
= & -p_{n} T_{m-1}(n-1) .
\end{aligned}
$$

[17] S. J. Lee and M. Gerla, "AODV-BR: Backup routing in ad hoc networks," in Proc. IEEE WCNC, 2000, pp. 1311-1316.

[18] C. E. Perkins and E. M. Royer, "Ad-hoc on-demand distance vector routing," in Proc. IEEE WMCSA, New Orleans, LA, Feb. 1999, pp. $90-100$

[19] L. Wang, L. Zhang, Y. Shu, and M. Dong, "Multipath source routing in wireless ad hoc networks," in Proc. Canadian Conf. Electrical and Computer Engineering, vol. 1, 2000, pp. 479-483.

[20] S. J. Lee and M. Gerla, "Split multipath routing with maximally disjoint paths in ad hoc networks," in Proc. ICC 2001, vol. 10, June 2001, pp. 3201-3205.

[21] P. Papadimitratos, Z. J. Haas, and E. G. Sirer, "Path set selection in mobile ad hoc networks," in Proc. ACM MobiHOC 2002, Lausanne, Switzerland, June 9-11, 2002, pp. 160-170.

[22] M. R. Pearlman, Z. J. Haas, P. Sholander, and S. S. Tabrizi, "On the impact of alternate path routing for load balancing in mobile ad hoc networks," in Proc. MobiHOC, 2000, pp. 150-310.

[23] N. Gogate, D. Chung, S. Panwar, and Y. Wang, "Supporting video/image applications in a mobile multihop radio environment using route diversity," in Proc. IEEE ICC '99, vol. 3, June 1999, pp. 1701-1706.

[24] A. Tsirigos and Z. J. Haas, Analysis of Multipath Routing: Part 2-Mitigation of the Effects of Frequently Changing Network Topologies, submitted for publication.

[25] A. B. McDonald and T. Znati, "A path availability model for wireless ad hoc networks," in Proc. IEEE WCNC, vol. 1, 1999, pp. 35-40.

[26] C.-K. Toh, "Associativity-based routing for ad hoc mobile networks," Wireless Pers. Commun. J., vol. 4, pp. 103-139, Mar. 1997.

[27] R. Dube, C. D. Rais, W. Kuang-Yeh, and S. K. Tripathi, "Signal stabilitybased adaptive routing (SSA) for ad hoc mobile networks," IEEE Pers. Commun., pp. 36-45, Feb. 1997.

\section{REFERENCES}

[1] N. F. Maxemchuk, "Dispersity routing," in Proc. IEEE ICC '75, June 1975, pp. 41.10-41.13.

[2] E. Ayanoglu, C.-L. I, R. D. Gitlin, and J. E. Mazo, "Diversity coding for transparent self-healing and fault-tolerant communication networks," IEEE Trans. Commun., vol. 41, pp. 1677-1686, Nov. 1993.

[3] R. Krishnan and J. A. Silvester, "Choice of allocation granularity in multipath source routing schemes," in Proc. IEEE INFOCOM '93, vol. 1, 1993, pp. 322-329.

[4] A. Banerjea, "Simulation study of the capacity effects of dispersity routing for fault-tolerant realtime channels," in Proc. ACM SIGCOMM'96, vol. 26, 1996, pp. 194-205.

[5] D. Sidhu, R. Nair, and S. Abdallah, "Finding disjoint paths in networks," in Proc. ACM SIGCOMM '91, 1991, pp. 43-51.

[6] R. Ogier, V. Rutemburg, and N. Shacham, "Distributed algorithms for computing shortest pairs of disjoint paths," IEEE Trans. Inform. Theory, vol. 39, pp. 443-455, Mar. 1993.

[7] S. Murthy and J. J. Garcia-Luna-Aceves, "Congestion-oriented shortest multipath routing," in Proc. IEEE INFOCOM '96, Mar. 1996, pp. 1028-1036.

[8] W. T. Zaumen and J. J. Garcia-Luna-Aceves, "Loop-free multipath routing using generalized diffusing computations," in Proc. IEEE INFOCOM '98, Mar. 1998, pp. 1408-1417.

[9] J. Chen, P. Druschel, and D. Subramanian, "An efficient multipath forwarding method," in Proc. IEEE INFOCOM '98, 1998, pp. 1418-1425.

[10] N. Taft-Plotkin, B. Bellur, and R. Ogier, "Quality-of-Service routing using maximally disjoint paths," in Proc. 7th Int. Workshop Quality of Service (IWQoS'99), June 1999, pp. 119-128.

[11] S. Vutukury and J. J. Garcia-Luna-Aceves, "An algorithm for multipath computation using distance vectors with predecessor information," in Proc. IEEE ICCCN '99, Oct. 1999, pp. 534-539.

[12] I. Cidon, R. Rom, and Y. Shavitt, "Analysis of multi-path routing," IEEE/ACM Trans. Networking, vol. 7, pp. 885-896, Dec. 1999.

[13] A. Nasipuri and S. R. Das, "On-demand multipath routing for mobile ad hoc networks," in Proc. IEEE ICCCN, Oct. 1999, pp. 64-70.

[14] D. Johnson and D. Maltz, "Dynamic source routing in ad hoc wireless networks," in Mobile Computing, T. Imielinski and H. Korth, Eds. Norwell, MA: Kluwer, 1996.

[15] V. D. Park and M. S. Corson, "A highly adaptive distributed routing algorithm for mobile wireless networks," in Proc. IEEE INFOCOM '99, 1999 , pp. $1405-1413$

[16] J. Raju and J. J. Garcia-Luna-Aceves, "A new approach to on-demand loop-free multipath routing," in Proc. IEEE ICCCN, Oct. 1999, pp. $522-527$.

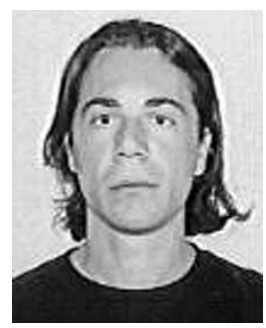

Aristotelis Tsirigos received the B.Sc. degree in electrical engineering and computer science from the National Technical University of Athens, Athens, Greece, in 1998, and the M.S. degree in electrical and computer engineering from Cornell University, Ithaca, NY, in 2001. Currently, he is working toward the Ph.D. degree in computational biology at New York University, New York, NY.

Since June 2003, he has been a member of the Bioinformatics and Pattern Discovery group at IBM's Computational Biology Center. His research interests include mobile networking and computational biology.

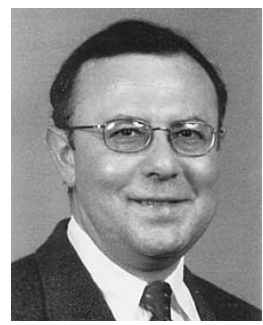

Zygmunt J. Haas (S'84-M'88-SM'90) received the B.Sc. degree in electrical engineering in 1979 and the M.Sc. degree in electrical engineering in 1985. In 1988, he received the Ph.D. degree from Stanford University, Stanford, CA.

He subsequently joined AT\&T Bell Laboratories in the Network Research Department. There he pursued research on wireless communications, mobility management, fast protocols, optical networks, and optical switching. From September 1994 till July 1995, was with the AT\&T Wireless Center of Excellence, where he investigated various aspects of wireless and mobile networking, concentrating on TCP/IP networks. As of August 1995, he joined the faculty of the School of Electrical and Computer Engineering, Cornell University, Ithaca, NY. He is an author of numerous technical papers and holds 15 patents in the fields of high-speed networking, wireless networks, and optical switching. His interests include mobile and wireless communication and networks, personal communication service, and high-speed communication and protocols.

Dr. Haas has organized several workshops, delivered numerous tutorials at major IEEE and ACM conferences, and serves as Editor of several journals and magazines, including the IEEE TRANSACTIONS ON NETWORKING, the IEEE TRANSACTIONS ON WIRELESS COMMUNICATIONS, the IEEE Communications Magazine, and the ACM/Kluwer Wireless Networks journal. He has been a Guest Editor of the IEEE JOURNAL ON SELECTED AREAS IN COMMUNICATIONS issues on gigabit networks, mobile computing networks, and ad-hoc networks. He is a voting member of ACM, and the Chair of the IEEE Technical Committee on Personal Communications. 